\title{
Métodos de quantificação de carga de treinamento em exercício realizado no máximo estado estável de lactato
}

\author{
Fábio Yuzo Nakamura ${ }^{1}$ \\ Lúcio Flávio Soares-Caldeira ${ }^{1}$ \\ Nilo Massaru Okuno² \\ Alexandre Moreira ${ }^{2}$ \\ Herbert Gustavo Simões ${ }^{3}$ \\ ${ }^{1}$ Universidade Estadual de Londrina, Londrina, PR, Brasil \\ ${ }^{2}$ Universidade de São Paulo, São Paulo, SP, Brasil \\ ${ }^{3}$ Universidade Católica de Brasília, Brasília, DF, Brasil
}

\begin{abstract}
Resumo: $O$ objetivo do presente estudo foi comparar e correlacionar as estimativas de impulso de

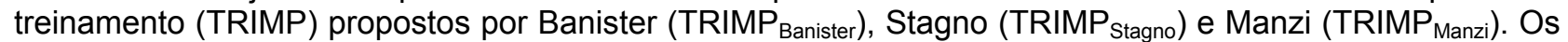
participantes foram submetidos a um teste progressivo em cicloergômetro, com registro da freqüência cardíaca e da concentração de lactato sanguíneo. Em uma segunda ocasião, realizaram 30 min. de exercício na intensidade correspondente ao máximo estado estável de lactato, a partir do qual foram calculados o TRIMP Banister, $_{\text {TRIMP }}$ Stagno e TRIMP $_{\text {Manzi }}$ Os valores médios de TRIMP Banister $(56,5 \pm 8,2$ u.a.) e $\operatorname{TRIMP}_{\text {Stagno }}(51,2 \pm 12,4$ u.a.) não diferiram entre si $(P>0,05)$ e foram altamente correlacionados $(r=0,90)$, com boa concordância, ou seja, viés reduzido e limites de concordância relativamente estreitos. $O$ TRIMP $_{\text {Stagno }}$ e TRIMP Manzi $_{\text {( }}(73,4 \pm 17,6$ u.a.) apresentarem alta correlação $(r=0,93)$, mas com diferença significantes entre eles; ainda, se mostraram pouco concordantes. As estimativas de TRIMP $\mathrm{B}_{\text {Banister }} \mathrm{e}$

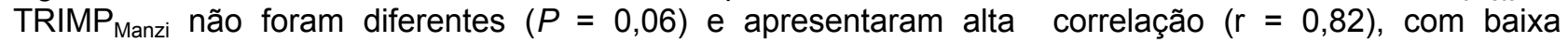
concordância. Assim, conclui-se que os métodos de TRIMP não são equivalentes. Na prática, parece ser prudente monitorar o processo de treinamento assumindo apenas uma das estimativas.
\end{abstract}

Palavras-chave: Impulso de treinamento. Freqüência cardíaca. Concentração de lactato. Máximo estado estável de lactato.

\section{Methods of quantification of training load in exercise performed at maximal lactate steady state}

\begin{abstract}
The aim of the present study was to compare and correlate training impulse (TRIMP) estimates proposed by Banister $\left(\right.$ TRIMP $\left._{\text {Banister }}\right)$, Stagno $\left(\right.$ TRIMP $\left._{\text {Stagno }}\right)$ and Manzi (TRIMP Manzi $_{1}$ ). The subjects were submitted to an incremental test on cycle ergometer with heart rate and blood lactate concentration measurements. In the second occasion, they performed $30 \mathrm{~min}$. of exercise at the intensity corresponding to maximal lactate steady state, and TRIMP $P_{\text {Banister, }}$ TRIMP $P_{\text {Stagno }}$ and TRIMP Manzi $_{\text {were calculated. The mean }}$ values of TRIMP Banister $\left(56.5 \pm 8.2\right.$ u.a.) and TRIMP ${ }_{\text {Stagno }}(51.2 \pm 12.4$ u.a.) were not different $(P>0.05)$ and were highly correlated $(r=0.90)$. Besides this, they presented a good agreement level, which means low bias and relatively narrow limits of agreement. On the other hand, despite highly correlated $(r=0.93)$, TRIMP $_{\text {Stagno }}$ and TRIMP ${ }_{\text {Manzi }}(73.4 \pm 17.6$ u.a.) were different $(P<0.05)$, with low agreement level. The

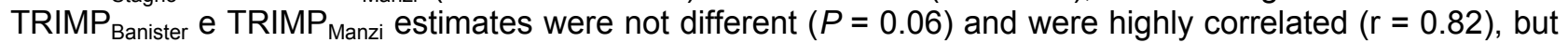
showed low agreement level. Thus, we concluded that the investigated TRIMP methods are not equivalent. In practical terms, it seems prudent monitor the training process assuming only one of the estimates.
\end{abstract}

Key Words: Training impulse. Heart rate. Blood lactate concentration. Maximal lactate steady state.

\section{Introdução}

Medidas de carga externa são obtidas na rotina do esporte para quantificar as cargas de treinamento. A velocidade de corrida e a distância de nado são exemplos de indicadores externos de intensidade e volume de treinamento, respectivamente. No entanto, cargas externas similares podem gerar níveis de estresse fisiológico distintos em diferentes atletas e, conseqüentemente, induzir respostas adaptativas variadas. Inversamente, cargas externas distintas podem ter repercussões fisiológicas agudas semelhantes. Dessa forma, evidencia-se a necessidade de se conhecer as cargas internas resultantes das sessões de treinamento para um monitoramento individual e eficaz do processo de treinamento. 
A carga interna é determinada pelas características inatas, nível inicial de aptidão física e natureza e quantidade de carga externa (IMPELLIZZERI et al., 2005). Uma das formas mais utilizadas em pesquisa para quantificar a carga interna baseia-se no cálculo do impulso de treinamento (TRIMP) (BANISTER; CALVERT, 1980). Esse método pressupõe que a fração de aumento da freqüência cardíaca de reserva $(\mathrm{FCr})$ durante o exercício, multiplicada pela duração da sessão do treinamento, constitui uma aproximação da carga interna. Com o intuito de ajustar esse valor para dar mais peso às cargas realizadas em intensidades mais altas, Banister (1991) sugeriu que o resultado fosse multiplicado por uma constante representativa do aumento exponencial da concentração de lactato sanguíneo ([La]) em função da fração de aumento da FCr. Dessa forma, para homens a equação tem o seguinte formato:

$\operatorname{TRIMP}_{\text {Banister }}=$ duração $\cdot \mathrm{FCr} \cdot 0,64 \cdot \mathrm{e}^{1,92 \mathrm{FCr}} \quad$ [equação 1]

Apesar de ter produzido resultados acurados de predição de desempenho (BUSSO et al., 1991; FITZ-CLARKE et al., 1991; MORTON et al., 1990), a equação de Banister (1991) pode não ser adequada para todos os indivíduos, uma vez que as curvas de lactato são bastante heterogêneas (HECK et al., 1985). Corroborando essa hipótese, Stagno et al. (2007) encontraram valores bastante distintos para a constante e para o expoente da função exponencial entre jogadores de hockey, comparados aos valores adotados por Banister (1991):

TRIMP $_{\text {Stagno }}=$ duração $. \mathrm{FCr} \cdot 0,1225 \cdot \mathrm{e}^{3,9434 \mathrm{FCr}} \quad$ [equação 2]

Essa grande variação nas respostas individuais de lactacidemia ao teste progressivo levou Manzi et al. (2009a,b) a proporem a utilização de curvas individuais para a estimativa do TRIMP (TRIMP Manzi). $_{\text {. }}$.

Levando em consideração que o construto da estimativa dos diferentes TRIMPs possui natureza distinta, o objetivo deste estudo foi comparar, correlacionar e verificar a concordância entre as estimativas de TRIMP $_{\text {Banister, }}$ TRIMP Stagno $_{\text {e TRIMP }}$ Manzi derivados de exercício realizado em intensidade correspondente ao máximo estado estável de lactato. Essa intensidade foi escolhida neste estudo por ser uma referência para prescrição de treinamento aeróbio em atletas e não atletas, em diferentes faixas etárias (BENEKE et al., 2009;
PARDONO et al., 2008). Dessa forma, a quantificação da carga interna em uma simulação de treinamento no máximo estado estável de lactato poderia fornecer parâmetros para técnicos e preparadores físicos que utilizam métodos baseados em TRIMP para a prescrição do treinamento.

Em função das diferentes suposições inerentes às equações de estimativa de TRIMP, poder-se-ia ter diferentes respostas decorrentes do uso de uma ou outra abordagem. Portanto, foi hipotetizado que os métodos apresentariam diferenças significantes e baixo nível de concordância, e que, portanto, não poderiam ser empregados como se fossem equivalentes.

\section{Sujeitos}

\section{Métodos}

Participaram deste estudo 8 homens jovens (idade: $25,3 \pm 4,5$ anos; massa corporal: $72,7 \pm$ $8,4 \mathrm{~kg}$; estatura: $177,0 \pm 5,0 \mathrm{~cm}$ ), fisicamente ativos. Após serem informados acerca dos procedimentos, riscos e benefícios associados à participação no estudo, assinaram um termo de consentimento livre esclarecido. Este estudo foi aprovado pelo Comitê de Ética em Pesquisa local (Processo $n^{\circ}$ 100/06).

Os participantes foram orientados a manter $\mathrm{O}$ padrão alimentar habitual, não realizar exercícios físicos intensos, e não ingerirem bebidas alcoólicas e cafeinadas dentro do intervalo de 24 $\mathrm{h}$ prévias aos testes. Além disso, foram orientados também a realizar uma refeição leve 3 $\mathrm{h}$ antes do início dos testes.

\section{Delineamento experimental}

Os participantes foram submetidos a um teste progressivo em cicloergômetro, com registro da fração de aumento da FCr e da [La]. Em dias separados, foram submetidos a quatro cargas preditivas para estimativa da potência crítica. A potência crítica foi adotada como referência para determinação do máximo estado estável de lactato (POOLE et al., 1988). Por fim, realizaram 30 min. de exercício na potência correspondente ao máximo estado estável de lactato, para cálculo do TRIMP a partir dos métodos propostos por Banister (1991) e Stagno et al. (2007), os quais se baseiam em curvas padronizadas de [La] vs. $\mathrm{FCr}$, e a partir do método de Manzi et al. (2009a,b), calculado a partir das curvas individuais. As estimativas de TRIMP Banister, TRIMP $_{\text {Stagno }}$ e TRIMP Manzi foram comparadas. Em 
adição, foi verificada a associação e a concordância entre elas.

\section{Teste progressivo}

O teste progressivo foi precedido por um período de aquecimento de 3 minutos realizado a 30 W em um cicloergômetro eletromagnético (Lode Excalibur, Groningen, Holanda). Para cada sujeito, a altura do selim foi ajustada individualmente para permitir que os joelhos atingissem extensão quase completa durante a revolução do pedal. Além disso, o apoio das mãos (guidão) foi ajustado para proporcionar conforto ao avaliado, sendo as configurações individuais mantidas constantes para todos os testes subseqüentes.

Após o aquecimento, os sujeitos começaram a pedalar a $70 \mathrm{rpm}$ contra uma carga inicial de 30 $\mathrm{W}$, com incrementos de $10 \mathrm{~W} \cdot \mathrm{min}^{-1}$, até que atingissem a exaustão voluntária. A maior potência atingida e mantida pela duração de um minuto no teste era considerada a máxima potência aeróbia (MAP). As análises de consumo de oxigênio $\left(\mathrm{VO}_{2}\right)$ foram realizadas respiração-arespiração (Cortex ${ }^{\circledR}$ MetaLyzer 3B, Alemanha). O aparelho de análise de gases foi calibrado antes de cada teste a partir do ar ambiente e por meio de um cilindro contendo concentrações conhecidas de $\mathrm{O}_{2}(16 \%)$ e $\mathrm{CO}_{2}(5 \%)$. O fluxo de ar foi calibrado utilizando uma seringa de três litros.

$\mathrm{O}$ consumo máximo de oxigênio $\left(\mathrm{VO}_{2} \max \right)$ foi considerado como a média dos valores de $\mathrm{VO}_{2}$ nos últimos 30 segundos de esforço. Para confirmar a ocorrência do $\mathrm{VO}_{2} \max$, deveriam ser observados pelo menos dois dos três critérios a seguir: (1) um platô no $\mathrm{VO}_{2}$; (2) razão de trocas respiratórias maior que 1,10; (3) freqüência cardíaca $(F C)$ maior que $90 \%$ do máximo predito para a idade. A FC em repouso e em exercício foi medida por meio de um cardiofrequencímetro Polar S810 (Polar ${ }^{\circledR}$, Finlândia)

Amostras sanguíneas $(25 \mu \mathrm{l})$ foram coletadas a partir do lóbulo da orelha a cada três minutos de teste. Cada amostra de sangue foi imediatamente transferida para túbulos de polietileno tipo eppendorff, contendo $50 \mu \mathrm{l}$ de fluoreto de sódio (NaF) a $1 \%$, sendo posteriormente armazenados em gelo. As análises das [La] foram realizadas utilizando-se um analisador eletroquímico modelo YSI 1500 Sport (Yellow Springs, Ohio, EUA).
Testes para determinação da potência crítica

A potência crítica foi determinada a partir da relação hiperbólica individual entre potência e tempo até exaustão em quatro cargas preditivas. Os testes eram realizados em dias diferentes. $\mathrm{O}$ aquecimento era o mesmo realizado previamente ao teste progressivo. Invariavelmente, o primeiro teste era realizado na MAP. Tomando por base a duração desse primeiro teste, mais três cargas preditivas tinham potências definidas em percentual da MAP de forma que suas durações variassem entre 1-15 minutos. A ordem de aplicação desses três testes era determinada por sorteio. A potência crítica corresponde a assíntota da relação hiperbólica entre potência e tempo até exaustão (MONOD; SCHERRER, 1965).

\section{Teste no máximo estado estável de lactato}

A intensidade correspondente ao máximo estado estável de lactato foi determinada a partir da realização de testes retangulares, até que o critério de aumento $<1 \mathrm{mM}$ de lactato fosse observado entre o $10^{\circ}$ e o $30^{\circ}$ minuto de exercício (HECK et al., 1985). A carga inicial testada para essa determinação foi a potência crítica. Quando a potência crítica superestimava o máximo estado estável de lactato, a carga era diminuída em $5 \%$. Em situação inversa, ou seja, observação de estabilização do lactato na potência crítica, a carga era aumentada em $5 \%$ até que o máximo estado estável fosse confirmado. Todos os participantes toleraram ao menos 30 minutos sustentando o máximo estado estável de lactato. Dessa forma, os cálculos de TRIMP foram feitas a partir da fração da FCr registrada a cada minuto dentro dessa duração fixa.

\section{Cálculo do impulso de treinamento}

O TRIMP Banister foi calculado a partir do registro da fração da FCr média observada ao longo do teste de máximo estado estável de lactato, aplicando-se a equação 1 . Os procedimentos de TRIMP $_{\text {Stagno }}$ e TRIMP Manzi, por outro lado, envolvem a estimativa de TRIMP momento a momento dentro da sessão, para então se efetuar a somatória. Optamos por determinar os valores minuto a minuto, pois no exercício aeróbio contínuo não há grande variação da $\mathrm{FC}$ como nos exercícios intermitentes. Os TRIMP stagno $\mathrm{e}$ TRIMP $_{\text {Manzi }}$ foram determinados a partir da 
somatória desses valores. O TRIMP Stagno foi calculado a partir da equação 2 , ao passo que o TRIMP $_{\text {Manzi }}$ foi calculado a partir de valores individuais obtidos para a constante e expoente no teste incremental, conforme exemplo na figura 1 , em que a constante foi de 0,2715 e o expoente de 3,36 . Os resultados dessa função foram multiplicados pela fração de aumento da $\mathrm{FCr}$ e pela duração da sessão, assim como nos métodos de Banister (1991) e Stagno et al. $\underline{(2007)}$.

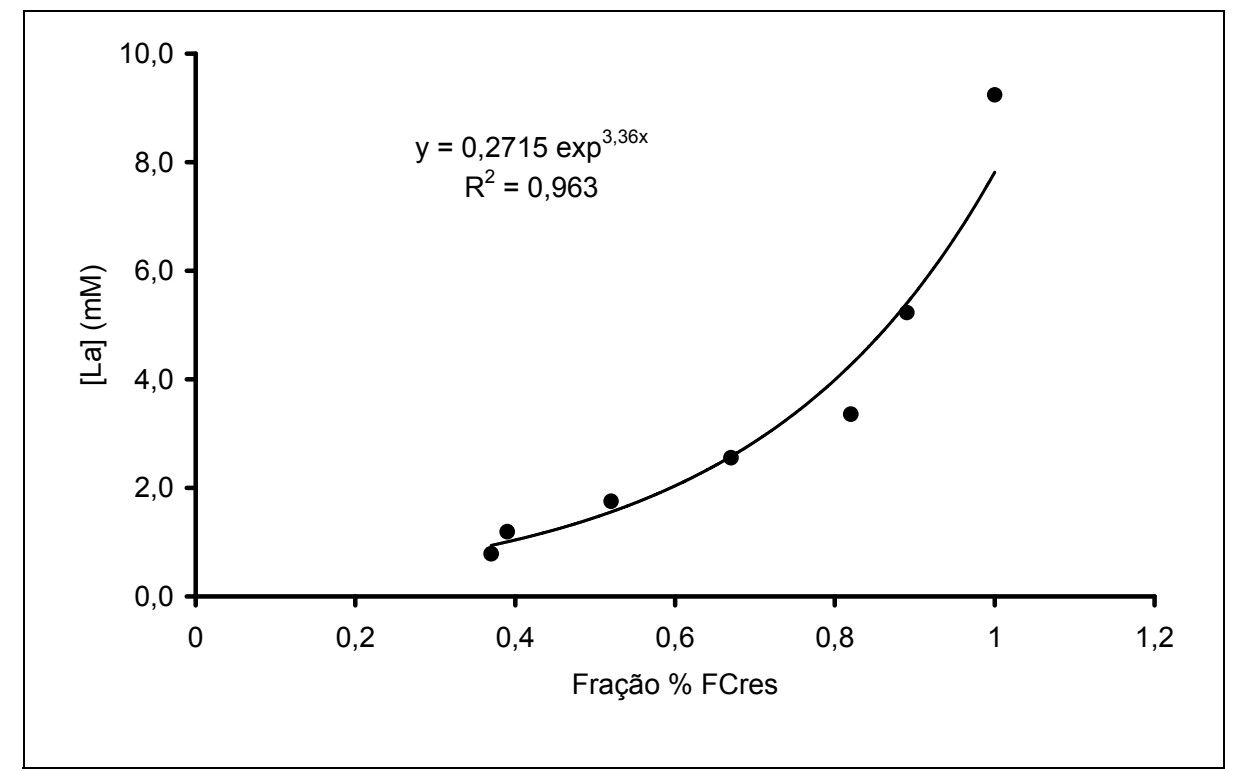

Figura 1. Exemplo de relação entre lactato sanguíneo e fração de aumento da freqüência cardíaca conforme método de Manzi et al. (2009).

\section{Análise estatística}

Os resultados são apresentados em média \pm desvio padrão. Os TRIMPs foram expressos em unidades arbitrárias (u.a.). A comparação entre as estimativas de TRIMP (TRIMP Banister, TRIMP $_{\text {Stagno }}$ e TRIMP $_{\text {Manzi }}$ ) foi feita a partir de ANOVA one-way para medidas repetidas, seguida do teste post hoc de Scheffé para identificação das diferenças. Neste caso, o teste de esfericidade de Mauchly foi aplicado previamente, confirmando que as estimativas de TRIMP atenderam ao pressuposto de ortonormalização. Além disso, a normalidade dos dados foi analisada por meio do teste de Kolmogorov-Smirnov, com correção de Lilliefors. Para verificar a associação entre as mesmas, bem como sua concordância, foram empregados correlação de Pearson e plotagem de BlandAltman (1986), respectivamente. A partir da plotagem de Bland-Altman (1986), que relaciona as médias individuais entre duas estimativas (ex. TRIMP $_{\text {Stagno }}$ e TRIMP Manzi $_{\text {) com as respectivas }}$ diferenças individuais, obtêm-se o viés (diferença média) e o intervalo de confiança (95\%) dentro do qual as diferenças ocorrem. Nas análises, foi adotado nível de significância de $5 \%$.

\section{Resultados}

O $\mathrm{VO}_{2} \max$ da amostra foi de 46,6 $\pm 5,8$ $\mathrm{ml} / \mathrm{kg} / \mathrm{min}$. Os valores médios de TRIMP Banister

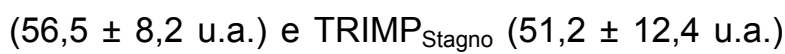
não diferiram entre si $(P>0,05)$. Além disso, eles se apresentaram altamente correlacionados $(r=$ 0,90; Figura 2A). A diferença média (viés) e os intervalos de confiança das diferenças individuais mostraram que as medidas eram relativamente concordantes (Tabela 1). Por outro lado, apesar de altamente correlacionados $(r=0,93$; Figura

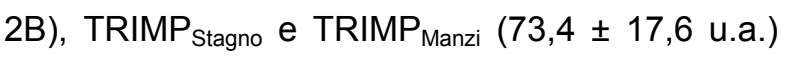
apresentaram diferença $(P<0,05)$ e se mostraram pouco concordantes (Tabela 1). As estimativas de TRIMP Banister $_{\text {e TRIMP }}$ Manzi se correlacionaram fortemente $(r=0,82$; Figura $2 \mathrm{C})$, não foram diferentes $(P=0,06)$, mas se mostraram pouco concordantes (Tabela 1). 


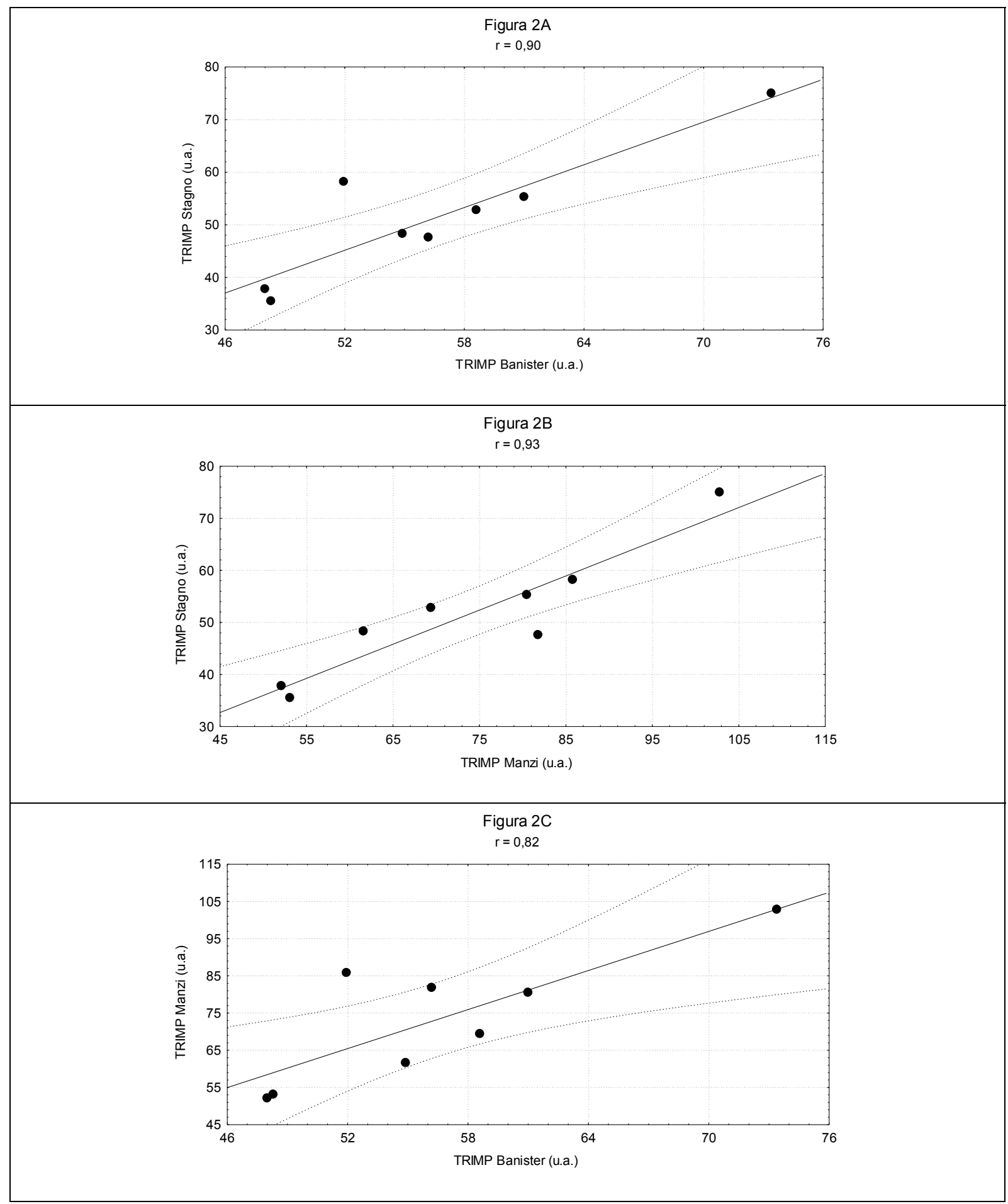

Figura 2. Correlações entre TRIMP obtidos a partir dos diferentes métodos.

\section{Discussão}

Os resultados do presente estudo mostraram que TRIMP Banister $_{\text {e TRIMP }}$ Stagno apresentam valores semelhantes e altamente correlacionados ao se quantificar a carga de treinamento resultante da manutenção de 30 minutos de exercício no máximo estado estável de lactato. No entanto, ambos tendem a subestimar os valores de carga de treinamento obtidos por meio de método baseado em curvas individuais de lactato pela fração de aumento da $\mathrm{FCr}$, denominada neste estudo de TRIMP Manzi. Esses $_{\text {. }}$ achados corroboram os reportados por Manzi et al. (2009b). Ainda assim, os métodos baseados em curvas padrão de lactato (TRIMP Banister $\mathrm{e}$ TRIMP $_{\text {Stagno }}$ ) se correlacionaram fortemente com 


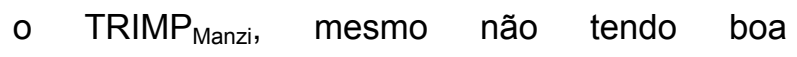
concordância com ele. Essa correlação não foi investigada por Manzi et al. (2009b). Neste estudo, Manzi et al. (2009b) investigaram a capacidade de TRIMP Banister e TRIMP $_{\text {Manzi }}$ em predizer mudanças no desempenho de corredores de endurance, e atribuíram a diferença entre essas estimativas ao fato do método de Banister et al. (1980) basear-se em FC média da sessão, e ao fato das constantes da curva exponencial não acompanharem as respostas fisiológicas individuais.

Diversos estudos têm proposto métodos de quantificação de cargas de treinamento no esporte, e testado sua validade e utilidade
(ALEXIOU; COUTTS, 2008; FOSTER et al., 2001; IMPELLIZZERI et al., 2004). Via de regra, o TRIMP $_{\text {Banister }}$ tem sido colocado como critério para validação concorrente de outros métodos, incluindo percepção subjetiva de esforço da sessão (NAKAMURA et al., 2009). No entanto, a proposição recente de outras equações (STAGNO et al., 2007), incluindo a individualização das cargas de treinamento (MANZI et al., 2009a,b), torna necessária a discussão mais aprofundada de qual método deve ser adotado como medida critério nesse tipo de investigação. Essa questão permanece não respondida, sendo $\circ$ TRIMP $_{\text {Banister }}$ ainda o mais adotado, provavelmente por ser o mais antigo.

Tabela 1. Resultados obtidos pela plotagem de Band-Altman entre as comparações dos métodos de determinação do TRIMP pelos métodos Banister, Stagno e Manzi.

\begin{tabular}{lccc}
\hline & Stagno vs. Banister & Manzi vs. Stagno & Manzi vs. Banister \\
\hline Viés & $-5,2$ & 22,1 & 16,8 \\
+ 2.DP & 7,0 & 37,0 & 40,0 \\
- 2.DP & $-17,5$ & 7,0 & $-6,3$ \\
IC & 24,4 & 44,2 & 46,3 \\
\hline
\end{tabular}

Viés = média das diferenças; $\mathrm{DP}$ = desvio padrão; IC = intervalo de confiança das diferenças.

$\mathrm{Na}$ tabela 2 são apresentados os resultados individuais da constante e expoente da relação entre aumento de [La] com a fração da FCr obtidas no teste incremental para determinação do TRIMP ${ }_{\text {Manzi. }}$

Tabela 2. Resultados individuais das constantes e expoentes da relação exponencial entre a taxa de aumento da [La] e fração da FCr no teste incremental para determinação do TRIMP ${ }_{\text {Manzi. }}$

\begin{tabular}{lll}
\hline \multicolumn{1}{c}{ Sujeito } & \multicolumn{1}{c}{ Equações } & $\mathbf{R}^{2}$ \\
\hline CH & $y=0,4421^{*} \exp ^{2,7829 x}$ & 0,95 \\
GU & $y=0,5084^{*} \exp ^{2,5184 x}$ & 0,88 \\
LU & $y=0,2416^{*} \exp ^{3,3606 x}$ & 0,95 \\
LF & $y=0,2715^{*} \exp ^{3,36 x}$ & 0,96 \\
MP & $y=0,2376{ }^{*} \exp ^{3,7783 x}$ & 0,99 \\
NO & $y=0,5266^{*} \exp ^{2,6153 x}$ & 0,98 \\
RC & $y=0,2014{ }^{*} \exp ^{3,7857 x}$ & 0,99 \\
SM & $y=0,1427{ }^{*} \exp ^{4,1401 x}$ & 0,95 \\
\hline
\end{tabular}

Apesar de se basearem em equações bastante diferentes, TRIMP Banister $_{\text {e TRIMP }}$ Stagno foram semelhantes, apresentaram boa concordância e foram altamente correlacionados. Isso pode ter ocorrido pelo fato do TRIMP Stagno $_{\text {ter }}$ sido estimado minuto a minuto, para depois ser somado ao final dos $30 \mathrm{~min}$. de exercício. Já o

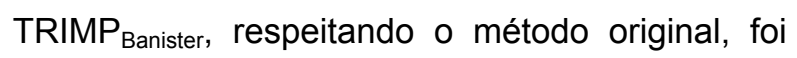
calculado a partir da média da elevação da $\mathrm{FCr}$ durante todo o exercício. Esse viés pode ter aproximado as duas estimativas, mesmo partido de equações bastante diferentes. No entanto, não se podem generalizar os resultados, pois é bastante provável que em situações de exercício intermitente ou estocástico, como por exemplo, a situação de competição e/ou treinamento nas modalidades esportivas coletivas, essa concordância entre as medidas seja reduzida. Uma vantagem de ser utilizar medidas minuto a minuto, ou até janelas menores (por exemplo, 5 segundos), é que o tempo mantido em diferentes faixas de intensidade pode ser quantificado (por exemplo, abaixo e acima dos limiares metabólicos; LUCIA et al., 2003; SEILER; KJERLAND, 2006). Portanto, sugerimos que mesmo ao se utilizar o método de Banister (1991), as estimativas de cargas de treinamento 
sejam obtidas em janelas menores de tempo, em detrimento da medida global ao final do treinamento. Isso pode auxiliar na quantificação do tempo mantido em diferentes faixas de intensidades.

Esse pressuposto é atendido pelo método proposto por Manzi et al. (2009a,b), que inclusive assume medidas de fração de elevação da $\mathrm{FCr}$ a cada 5 segundos. No presente estudo, optamos por medidas a cada minuto pelo fato do exercício ter sido realizado contra carga fixa, tendo reduzidas oscilações da resposta fisiológica. Além disso, diferentemente de TRIMP Banister e TRIMP $_{\text {Stagno, o TRIMP }}$ Manzi leva em consideração as respostas individuais de lactato vs. aumento da FCr. A partir da tabela 2, pode-se verificar que há considerável variabilidade inter-individual nos valores da constante e do expoente da função exponencial que descreve o aumento da [La]. Dessa forma, torna-se questionável o uso de curvas fixas, conforme métodos de Banister (1991) e Stagno et al. (2007). Além disso, devese lembrar que o treinamento pode modificar as curvas de lactato em função da carga externa (MARCELL et al., 2003), e possivelmente pode causar alterações na relação entre lactato e fração de aumento da FCr.

O método de TRIMP Manzi gerou valores maiores de carga de treinamento em relação a

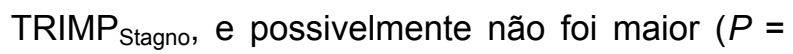

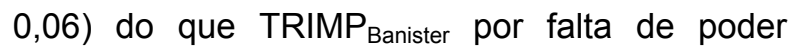
estatístico, dado o número reduzido de participantes. Além disso, as medidas de TRIMP $_{\text {Manzi }}$ foram pouco concordantes com relação aos métodos baseados em curvas fixas de lactato. Assim, pode-se considerar que os métodos não sejam equivalentes. Há evidências de que TRIMP $_{\text {Manzi }}$ seja superior a TRIMP Banister quanto à eficácia, pois o primeiro está mais bem relacionado a mudanças no desempenho (MANZI et al., 2009b).

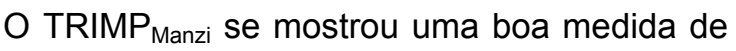
carga de treinamento ao se estabelecer a relação dose-resposta entre esta e a mudança em indicadores autonômicos, que por sua vez foram preditores de desempenho na maratona (MANZI et al., 2009a). Dessa forma, sugere-se que mais estudos sejam conduzidos a partir deste método, assumindo que ele produz valores maiores de TRIMP e com maior relação com o melhora do desempenho (MANZl et al., 2009b) do que os métodos baseados em curvas fixas de lactato.
Ainda assim, como os métodos são altamente correlacionados, é possível que para algumas finalidades, como por exemplo, construção de curvas de periodização, todos eles possam ser utilizados, uma vez que TRIMP Banister e TRIMP $_{\text {Stagno também se mostraram bons }}$ preditores de mudança de desempenho em estudos realizados previamente (BUSSO et al., 1991; FITZ-CLARKE et al., 1991; MORTON et al., 1990; STAGNO et al., 2007). Estudos comparativos para predição de desempenho, como o conduzido por Manzi et al. (2009b) devem também ser realizados como forma de estabelecer se há de fato superioridade do método individual no monitoramento das respostas ao treinamento.

Assim, conclui-se que os métodos de TRIMP não podem ser empregados de forma a serem considerados equivalentes, considerando que quando o método de curva individual da relação

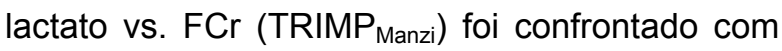
os métodos de TRIMP $_{\text {Banister e }}$ TRIMP $_{\text {Stagno, }}$ diferença significativa e/ou baixa concordância foram observadas. Vale ressaltar, no entanto, que as estimativas fornecidas pelos diferentes métodos foram altamente correlacionadas. Isso pode sugerir que na prática do treinamento esportivo, ao menos para situações onde as cargas são relativamente constantes ao longo da sessão, os métodos podem gerar padrões de curva de cargas de treinamento (periodização) semelhantes; no entanto, aconselhamos o método individual em função de ele incorporar possíveis alterações dos parâmetros das curvas de lactato e $\mathrm{FCr}$ ao longo da temporada. Essa questão deve ser levantada em estudos futuros que comparem métodos de quantificação de treinamento.

\section{Referências}

ALEXIOU, H.; COUTTS, A.J. A comparison of methods used for quantifying internal training load in women soccer players. International Journal of Sports Physiology and Performance, v. 3 , n.3, p.320-330, 2008. Disponível em: http://hk.humankinetics.com/IJSPP/viewarticle.cf m?jid=XhUcQX8xXxDrGE8EXvBtBJHQXpDpZJA 8XgNmYD\&aid=16197\&site=XhUcQX8xXxDrGE8 EXvBtBJHQXpDpZJA8XgNmYD. Acesso em: 11 out. 2009

BANISTER, E.W.; CALVERT, T.W. Planning for future performance: implications for long term training. Canadian Journal of Applied Sport Sciences, v.5, n.3, p.170-176, 1980. 
BANISTER, E.W. Modeling elite athletic performance. In: GREEN, H.; MCDOUGAL, J.; WENGER, H. Physiological Testing of Elite Athletes. Ed. Human Kinetics, Champaign, 1991. p.403-424.

BENEKE, R.; HECK, H.; HEBESTREIT, H.; LEITHÄUSER, R.M. Predicting maximal lactate steady state in children and adults. Pediatric Exercise Science, v.21, n.4, p.493-505, 2009. Disponível em:

http://hk.humankinetics.com/PES/viewarticle.cfm? jid=D7p6JhZEF7j2ZdqCE3u6MuW4R7w7TusaC2 c6KtJzQ2e\&aid=17633\&site=D7p6JhZEF7j2ZdqC E3u6MuW4R7w7TusaC2c6KtJzQ2e. Acesso em: 10 fev. 2010.

BLAND, J.M.; ALTMAN, D.G. Statistical methods for assessing agreement between two methods of clinical measurement. Lancet, v.1, n.8476, p.307310, 1986. http://dx.doi.org/10.1016/S01406736(86)90837-8

BUSSO, T.; CARASSO, C.; LACOUR, J.R. Adequacy of a systems structure in the modeling of training effects on performance. Journal of Applied Physiology, v.71, n.5, p.2044-2049, 1991. Disponível em:

http://jap.physiology.org/cgi/content/abstract/71/5/ 2044. Acesso em: 11 out. 2009.

FITZ-CLARKE, J.R.; MORTON, R.H.; BANISTER, E.W. Optimizing athletic performance by influence curves. Journal of Applied Physiology, v.71, n.3, p.1151-1158, 1991. Disponível em: http://jap.physiology.org/cgi/content/abstract/71/3/ 1151. Acesso em: 11 out. 2009.

FOSTER, C.; FLORHAUG, J.A.; FRANKLIN, J.; GOTTSCHALL, L.; HROVATIN, L.A.; PARKER, S.; DOLESHAL, P.; DODGE, C. A new approach to monitoring exercise training. The Journal of Strength and Conditioning Research, v.15, n.1, p.109-115, 2001. Disponível em:

http://journals.Iww.com/nsca-

iscr/Abstract/2001/02000/A New Approach to M onitoring Exercise Training.19.aspx. Acesso em 11 out. 2009.

HECK, H.; MADER, A.; HESSE, G.; MUCKE, S.; MULLER, R.; HOLLMAN, W. Justification of the 4$\mathrm{mmol} / \mathrm{l}$ lactate threshold. International Journal of Sports Medicine, v.6, n.3, p.117-130, 1985.

Disponível em: http://www.thiemeconnect.de/ejournals/toc/sportsmed/30098. Acesso em: 11 out. 2009.

IMPELLIZZERI, F.M.; RAMPININI, E.; COUTTS, A.J.; SASSI, A.; MARCORA, S.M. Use of RPEbased training load in soccer. Medicine and Science in Sports and Exercise, v.36, n.6, p.1042-1047, 2004. Disponível em:

http://journals.Iww.com/acsmmsse/Abstract/2004/06000/Use of RPE Based
Training Load in Soccer.18.aspx. Acesso em: 11 out. 2009.

IMPELLIZZERI, F.M.; RAMPININI, E.; MARCORA, S.M. Physiological assessment of aerobic training in soccer. Journal of Sports Sciences, v.23, n.6, p.583-592, 2005. http://dx.doi.org/10.1080/02640410400021278

LUCIA, A.; HOYOS, J.; SANTALLA, A.; EARNEST, C.; CHICHARRO, J.L. Tour de France versus Vuelta a Espana: Which is harder? Medicine and Science in Sports and Exercise, v.35, n.5, p.872-878, 2003. Disponível em: http://journals.lww.com/acsmmsse/Abstract/2003/05000/Tour de France vers us Vuelta a Espana Which Is.23.aspx. Acesso em: 11 out. 2009.

MANZI, V.; CASTAGNA, C.; PADUA, E.; LOMBARDO, M.; D'OTTAVIO, S.; MASSARO, M.; VOLTERRANI, M.; IELLAMO, F. Dose-response relationship of autonomic nervous system responses to individualized training impulse in marathon runners. American Journal of Physiology - Heart and Circulatory Physiology, v.296, n.6, p.H1733-40, 2009a.

http://dx.doi.org/10.1152/ajpheart.00054.2009

MANZI, V.; IELLAMO, F.; IMPELLIZZERI, F.; D'OTTAVIO, S.; CASTAGNA, C. Relation between Individualized Training Impulses and Performance in Distance Runners. Medicine and Science in Sports and Exercise, 2009b. Disponível em: http://journals.Iww.com/acsmmsse/Abstract/publishahead/Relation between In dividualized Training Impulses.99498.aspx. Acesso em: 11 out. 2009.

MARCELL, T.J.; HAWKINS, S.A.; TARPENNING, K.M.; HYSLOP, D.M.; WISWELL, R.A.

Longitudinal analysis of lactate threshold in male and female master athletes. Medicine and Science in Sports and Exercise, v.35, n.5, p.810-817, 2003. Disponível em:

http://journals.lww.com/acsmmsse/Abstract/2003/05000/Longitudinal Analysis of Lactate Threshold in Male.14.aspx. Acesso em: 11 out. 2009

MONOD, H.; SCHERRER, J. The work capacity of a synergic muscular group. Ergonomics, v.8, p.329-338, 1965.

MORTON, R.H.; FITZ-CLARKE, J.R.; BANISTER, E.W. Modeling human performance in running. Journal of Applied Physiology, v.69, n.3, p.1171-1177, 1990. Disponível em: http://jap.physiology.org/cgi/content/abstract/69/3/ 1171. Acesso em: 11 out. 2009.

NAKAMURA, F.Y.; MOREIRA, A.; AOKI, M.S. Monitoramento da carga de treinamento: a percepção subjetiva do esforço da sessão é um 
método confiável? Revista da Educação Física, 2009.

PARDONO, E.; SOTERO, R.C.; HIYANE, W.; MOTA, M.R.; CAMPBELL, C.S.; NAKAMURA, F.Y.; SIMÕES, H.G. Maximal lactate steady-state prediction through quadratic modeling of selected stages of the lactate minimum test. The Journal of Strength and Conditioning Research, v.22, n.4, p.1073-1080, 2008.

http://journals.Iww.com/nscajscr/Abstract/2008/07000/Maximal Lactate Stead y State Prediction Through.7.aspx. Acesso em: 10 fev. 2010.

POOLE, D.C.; WARD, S.A.; GARDNER, G.W.; WHIPP, B.J. Metabolic and respiratory profile of the upper limit for prolonged exercise in man. Ergonomics, v.31, n.9, p.1265-1279, 1988.

STAGNO, K.M.; THATCHER, R.; VAN SOMEREN, K.A. A modified TRIMP to quantify the in-season training load of team sport players. Journal of Sports Sciences, v.25, n.6, p.629634, 2007.

http://dx.doi.org/10.1080/02640410600811817

SEILER, K.S.; KJERLAND, G.O. Quantifying training intensity distribution in elite endurance athletes: is there evidence for an "optimal" distribution? Scandinavian Journal of Medicine and Science in Sports, v.16, n.1, p.49-56, 2006. http://dx.doi.org/10.1111/j.1600$\underline{0838.2004 .00418 . x}$

\section{Endereço:}

Fábio Yuzo Nakamura

GEAFITCentro de Educação Física e Esporte

Departamento de Educação Física - Universidade Estadual de Londrina

Rodovia Celso Garcia Cid, km. 380

Campus Universitário

Londrina PR Brasil

86015-990

e-mail: fabioy_nakamura@yahoo.com.br

Recebido em: 26 de outubro de 2009.

Aceito em: 22 de fevereiro de 2010.

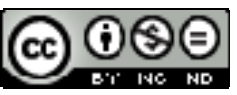

Motriz. Revista de Educação Física. UNESP, Rio Claro, SP, Brasil - elSSN: 1980-6574 - está licenciada sob Licenca Creative Commons 